myeloma: meta-analysis of trials with biological assignment. Bone Marrow Transplant 2013; 48: 562-567.

9 Network NCC. NCCN clinical practice guidelines in oncology (NCCN Guidelines). Multiple myeloma. Version 3. 2016. Available at: http://www.nccn.org/profes sionals/physician_gls/pdf/myeloma.pdf (accessed 24 May 2016).

10 Kronke J, Udeshi ND, Narla A, Grauman P, Hurst SN, McConkey M et al. Lenalidomide causes selective degradation of IKZF1 and IKZF3 in multiple myeloma cells. Science 2014; 343: 301-305.

11 Lu G, Middleton RE, Sun H, Naniong M, Ott CJ, Mitsiades CS et al. The myeloma drug lenalidomide promotes the cereblon-dependent destruction of Ikaros proteins. Science 2014; 343: 305-309.

12 Eichner R, Heider M, Fernandez-Saiz V, van Bebber F, Garz AK, Lemeer S et al. Immunomodulatory drugs disrupt the cereblon-CD147-MCT1 axis to exert antitumor activity and teratogenicity. Nat Med 2016; 22: 735-743.
13 Krönke J, Kuchenbauer F, Kull M, Teleanu V, Bullinger L, Bunjes D et al. IKZF1 expression is a prognostic marker in newly diagnosed standard-risk multiple myeloma treated with lenalidomide and intensive chemotherapy: a study of the German Myeloma Study Group (DSMM). Leukemia 2017; 31: 1363-1367.

14 Cavo M, Tosi P, Zamagni E, Cellini C, Tacchetti P, Patriarca F et al. Prospective, randomized study of single compared with double autologous stem-cell transplantation for multiple myeloma: Bologna 96 clinical study. J Clin Oncol 2007; 25: 2434-2441.

15 Knop S, Gerecke C, Liebisch P, Topp MS, Platzbecker U, Sezer O et al. Lenalidomide, adriamycin, and dexamethasone (RAD) in patients with relapsed and refractory multiple myeloma: a report from the German Myeloma Study Group DSMM (Deutsche Studiengruppe Multiples Myelom). Blood 2009; 113: 4137-4143.

\title{
OPEN
}

\section{Possible role of pandemic AH1N1 swine flu virus in a childhood leukemia cluster}

Leukemia (2017) 31, 1819-1821; doi:10.1038/leu.2017.127

The causation of acute lymphoblastic leukemia (ALL), the main subtype of childhood cancer, is still unresolved and the difficulty in uncovering a likely multi-factorial etiological pathway is compounded by biological heterogeneity of what is a relatively rare disease. ${ }^{1,2}$

There is persuasive evidence that the major subset of ALL, B-cell precursor or common ALL, is usually initiated pre-natally, in utero and that additional secondary genetic changes more proximal to diagnosis are required to trigger overt disease. ${ }^{2}$ Any putative etiologic mechanism has to accommodate this pattern of natural history. Among many candidate exposures, infection has long been regarded as a plausible causal agent for childhood $\mathrm{ALL}^{3,4}$ though no specific microbial infections or transforming viruses have been identified. ${ }^{2,3}$ Childhood ALL does not clearly cluster geographically, ${ }^{3}$ but this is not surprising if, as postulated, ALL causation involves a rare response to, or outcome of, a common infection of low or modest pathogenicity. ${ }^{2-4}$ There are, however, examples of what appear to be significant time/space clusters. ${ }^{5,6}$ Historically, the first of these (in 1957-1960) was in Niles, a suburb of Chicago, ${ }^{5}$ the most recent (1999-2004), and striking, was in Fallon, Nevada. ${ }^{6}$

We record here a time/space cluster of childhood B-cell precursor ALL in Milan, Italy. The seven cases involved had different ages (2-11 years), but were diagnosed within a tight time window of only 4 weeks (14 December 2009-14 January 2010); three cases being associated with a single school and a fourth case being resident in the same area as the school. This led us to suspect that there might be an identifiable trigger or promoting agent for ALL in this particular instance, to which all cases were exposed and which occurred very proximal to the diagnostic time period.

The main biological features of the patients at disease presentation are shown in Supplementary Table 1. All were 'common' B-cell precursor (CD10 positive) ALL, five female and two male, with a median age of 7.1 (range 2.1-11.9). Cytogenetic, molecular genetic and genomic alterations were variable but common to those generally present in ALL. ${ }^{1}$
We tested remission DNA of the patients for inherited or constitutive allelic variation in three genes associated with increased risk of B-cell precursor ALL: ARID5B, IKZF1 and CEBPE. ${ }^{7}$ The number of risk alleles present per case varied from one to five (out of six possible; average three) but does not differ from that expected (Supplementary Table 1).

Figure 1 shows the monthly distribution of cases diagnosed with childhood ALL between January 1999 and September 2011 in Milan; the broad-based peak, determined by two consecutive high frequencies observed in December 2009 (three cases) and January 2010 (four cases), has no equivalent in the considered period of 13 years (see also Supplementary Tables 2 and 3).

Figure 2 shows the results of the Scan statistic for the spacetime analysis of ALL cases. The Kulldorff's scan method ${ }^{8}$ identified only one significant cluster encompassing six administrative areas in the center of Milan within the time interval from 1 December 2009 to 31 January $2010(P$-value $=0.017)$ (see also Supplementary Table 4). The cluster is composed of four cases, compared with 0.04 expected, corresponding to a rate ratio of $100.0(95 \% \mathrm{Cl}$ : 26.9-256.0) for the areas and time period identified. The cases are exactly the four children resident in the surroundings of the school, three of them attending the school.

Because of long-lasting refurbishment, the primary school was not in operation until September 2009; in the previous year, the entire school population was temporarily hosted in the buildings of another school in the same city area. The concentration of all tested physical and chemical agents in the school as well as in the houses of cases did not deviate from normal ranges. Neither parents nor children had noticeable therapeutic exposures to postulated toxicants. None of the parents had professional exposures relevant for leukemia etiology. None of the cases had first-level familial malignancies. (Methods and details in Supplementary).

There was an outbreak of pandemic AH1N1 swine flu in Milan between July and September 2009 preceding the ALL diagnosis by around 3 to 6 months. Serological screening of plasma antibodies retrieved from blood smears at the time of ALL diagnosis (but before instigation of treatment) revealed that all seven were seropositive (titer range: 160-640; see Supplementary Table 5). In plasma samples taken in the same time period from 192 healthy 


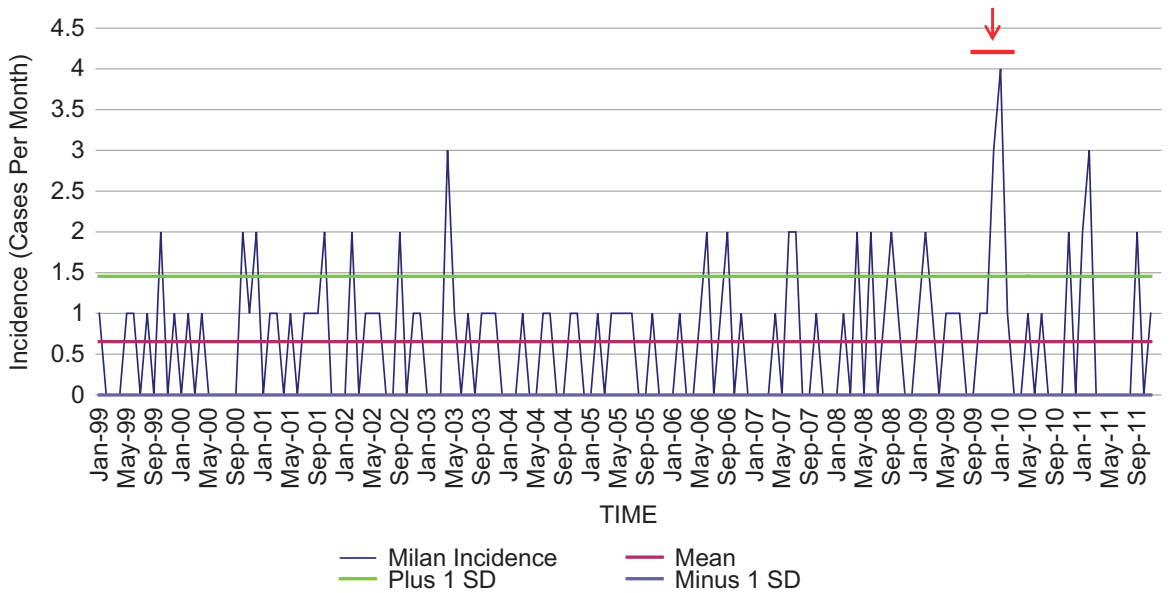

Figure 1. Monthly distribution of the children diagnosed with ALL between January 1999 and September 2011 in Milan, Italy. Mean incidence and \pm 1 s.d. The arrow indicates the time period in object.

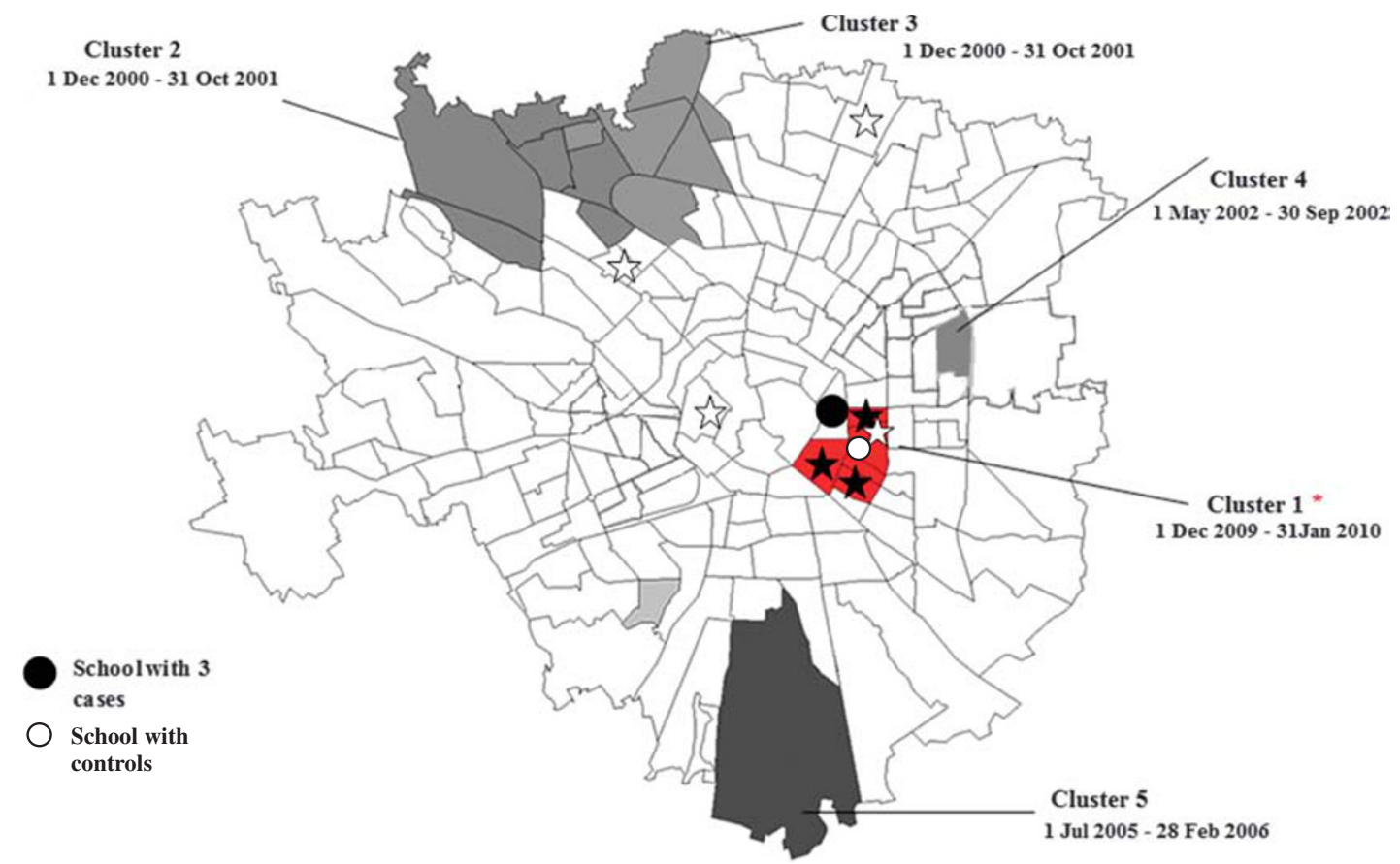

Figure 2. Representation of most likely spatial ALL clusters identified with Scan statistic in Milan, 1999-2010, considering a monthly time interval. The only statistically significant spatial cluster is in red, whereas different shades of gray identify non-statistically significant spatial clusters, with specification of corresponding time interval. The site of residence of leukemia cases is indicated by stars (black stars represent the three cases attending the same school). The location of the school of cases is represented by a black dot, whereas the open dot indicates the school where cases were temporarily hosted and where controls were recruited.

children from Milan, 62 (32.3\%) were seropositive suggesting the $100 \%$ positivity of the seven cases was significant $(P<0.001)$. Five cases of ALL diagnosed before the outbreak were, as expected, all seronegative. Of four solid pediatric tumors (Wilm's) diagnosed in Milan following the outbreak, only one was seropositive.

If abnormal immune responses to the widespread pandemic of AH1N1 served as a proximal trigger for ALL in the seven cases, then these cases would have to be 'susceptibles'. The 'delayed infection' hypothesis ${ }^{2}$ predicts that B-cell precursor ALL may be triggered by immune response signals but that risk is contingent upon deficits of common infections in infancy that are required to prime the neonatal and infant immune system. This is endorsed by measurement of social contacts in infancy as surrogates for common infections ${ }^{9-11}$ and by modeling in mice. ${ }^{12,13}$
We therefore investigated these characteristics in the seven families involved in the cluster. These were compared with a small series of control cases (Supplementary Table 6). Six out of seven cases were first born compared with nine out of 14 for the controls. All seven cases had parents of medium to high socioeconomic class compared with only two of the 20 controls. None of the seven cases attended day care in the first year of life (except for just 1 week in one case). Two of the seven cases attended day care in the $2-3$ years of life compared with 8 out of 14 controls. Of note is the related fact that the school with three cases serves an inner area of Milan of high socio-economic status professional parents.

A plausible explanation for the time/space cluster in Milan is that the AH1N1 epidemic provided a strong immunological or 
inflammatory signal that triggered conversion to clinical ALL in a small number of individuals harboring clinically silent preleukemic clones but who also were at risk of abnormal response to AH1N1 because of a prior deficit of microbial exposures in infancy that prime or modulate the naive immune system network. ${ }^{2}$

However, the causal explanation for the cluster we propose is based on associations and we cannot formally exclude a role for chance alone. It is therefore tentative. We encourage other investigators to scrutinize incidence data, both nationally and at local levels or within school systems, to see if other increases in incidence occurred shortly after the 2009 pandemic of AH1N1.

We do not suggest that $\mathrm{AH} 1 \mathrm{~N} 1$ has a unique etiological role in childhood ALL or that it poses a public health concern. ALL will, at most, be an extremely rare consequence of $\mathrm{AH} 1 \mathrm{~N} 1$ infection as part of a multi-factorial etiology. We would anticipate that other rare space/time clusters of childhood ALL may also be due to a promotional effect of infectious agents but not to AH1N1. Influenza viruses may, however, merit further study as indirect, promotional agents for ALL. A prior study noted an association between epidemics of seasonal influenza in the UK and significant increases in the incidence of ALL that follow very shortly ( 6 months) thereafter. ${ }^{14} \mathrm{AH} 1 \mathrm{~N} 1$, other influenza viruses and, possibly, other viral or bacterial infections may act as triggers or promoters of ALL.

\section{CONFLICT OF INTEREST}

The authors declare no conflict of interest.

\section{ACKNOWLEDGEMENTS}

This work was partially funded by grants from Fondazione Tettamanti, Comitato Fiori di Lavanda, Fondazione Cariplo, AIRC, Associazione Bianca Garavaglia Onlus, Bloodwise and The Institute of Cancer Research, London.

\section{ETHICS APPROVAL}

Written informed consent has been obtained from patients' guardians; this includes, without limitation, publication in all formats.

\section{AUTHOR CONTRIBUTIONS}

GC supervised the biological studies, contributed in collection, analysis and interpretation of the data, as well as in co-writing the paper. LB supervised the comprehensive investigation plan and the cluster analyses, contributed in collecting and interpreting data, and in writing the paper. GR and SD participated to the investigation plan and performed the cluster analyses. SB performed the biological analyses. FP provided the virological analyses and data. DP and FS provided control samples and data. GM, MGV and AB participated in the conception of the study and in interpretation of the data. MG supervised the whole project, provided the concept of the study, interpreted the data and co-wrote the manuscript. The corresponding author confirms that he had full access to all the data in the study and had final responsibility for the decision to submit for publication.

G Cazzaniga $^{1,9}$, L Bisanti ${ }^{2,9}$, G Randi ${ }^{2,10}$, S Deandrea ${ }^{2,10}$, S Bungaro ${ }^{1}$ F Pregliasco $^{3}$, D Perotti ${ }^{4}$, F Spreafico ${ }^{5}$, G Masera ${ }^{6}$, MG Valsecchi ${ }^{\prime}$, A Biondi ${ }^{6}$ and $M$ Greaves ${ }^{8}$

${ }^{1}$ Centro Ricerca Tettamanti, Clinica Pediatrica Università di Milano Bicocca, Fondazione MBBM/Ospedale San Gerardo, Monza, Italy; ${ }^{2}$ Servizio di Epidemiologia, Azienda Sanitaria Locale di Milano, Milano, Italy;

${ }^{3}$ Dipartimento di Scienze Biomediche per la Salute - Università degli Studi di Milano, Milano, Italy;
${ }^{4}$ Department of Preventive and Predictive Medicine, Unit of Molecular Bases of Genetic Risk and Genetic Testing, Fondazione IRCCS Istituto Nazionale dei Tumori, Milano, Italy;

${ }^{5}$ Pediatric Oncology Unit, Hematology and Pediatric Onco-Hematology Department, Fondazione IRCCS Istituto Nazionale dei Tumori, Milano, Italy;

${ }^{6}$ Clinica Pediatrica Università di Milano Bicocca, Fondazione MBBM/ Ospedale San Gerardo, Monza, Italy;

${ }^{7}$ Center of Biostatistics for Clinical Epidemiology, Department of Health Sciences, University of Milano-Bicocca, Monza, Italy and ${ }^{8}$ Centre for Evolution and Cancer, The Institute of Cancer Research, London, UK

E-mail: mel.greaves@icr.ac.uk ${ }^{9}$ These authors contributed equally to this work.

${ }^{10}$ Current address: European Commission - Directorate General Joint Research Centre; Directorate F-Health, Consumers and Reference Materials; Unit F1 'Health in Society', Ispra (VA), Italy.

\section{REFERENCES}

1 Inaba H, Greaves M, Mullighan CG. Acute lymphoblastic leukaemia. Lancet 2013; 381: 1943-1955.

2 Greaves M. Infection, immune responses and the aetiology of childhood leukaemia. Nat Rev Cancer 2006; 6: 193-203.

3 McNally RJQ, Eden TOB. An infectious aetiology for childhood acute leukaemia: a review of the evidence. Br J Haematol 2004; 127: 243-263.

4 Kinlen LJ. Epidemiological evidence for an infective basis in childhood leukaemia. Br J Cancer 1995; 71: 1-5.

5 Heath CW Jr, Hasterlik RJ. Leukemia among children in a suburban community. Am J Med 1963; 34: 796-812.

6 Francis SS, Selvin S, Yang W, Buffler PA, Wiemels JL. Unusual space-time patterning of the fallon, Nevada leukemia cluster: evidence of an infectious etiology. Chem Biol Interact 2012; 196: 102-109.

7 Prasad R, Hosking FJ, Vijayakrishnan J, Papaemmanuil E, Koehler R, Greaves M et al. Verification of the susceptibility loci on $7 p 12.2,10 q 21.2$ and $14 q 11.2$ in precursor B-cell acute lymphoblastic leukemia of childhood. Blood 2010; 115: 1765-1767.

8 Kulldorff M, Nagarwalla N. Spatial disease clusters: detection and inference. Stat Med 1995; 14: 799-810.

9 Urayama KY, Buffler PA, Gallagher ER, Ayoob JM, Ma X. A meta-analysis of the association between day-care attendance and childhood acute lymphoblastic leukaemia. Int J Epidemiol 2010; 39: 718-732.

10 Rudant J, Lightfoot T, Urayama KY, Petridou E, Dockerty JD, Magnani C et al. Childhood acute lymphoblastic leukemia and indicators of early immune stimulation: a Childhood Leukemia International Consortium study. Am J Epidemiol 2015; 181: 549-562.

11 Dockerty JD, Draper G, Vincent T, Rowan SD, Bunch KJ. Case-control study of parental age, parity and socioeconomic level in relation to childhood cancers. Int $J$ Epidemiol 2001; 30: 1428-1437.

12 Swaminathan S, Klemm L, Park E, Papaemmanuil E, Ford A, Kweon SM et al. Mechanisms of clonal evolution in childhood acute lymphoblastic leukemia. Nat Immunol 2015; 16: 766-774.

13 Martin-Lorenzo A, Hauer J, Vicente-Duenas C, Auer F, Gonzalez-Herrero I, GarciaRamirez l et al. Infection exposure is a causal factor in B-cell precursor acute lymphoblastic leukemia as a result of Pax5-inherited susceptibility. Cancer Discov 2015; 5: 1328-1343.

14 Kroll ME, Draper GJ, Stiller CA, Murphy MFG. Childhood leukemia incidence in Britain, 1974-2000: time trends and possible relation to influenza epidemics. J Natl Cancer Inst 2006; 98: 417-420.

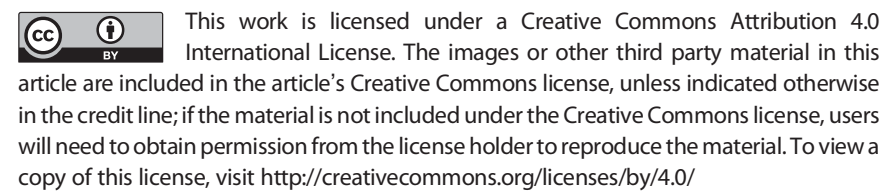

(c) The Author(s) 2017

Supplementary Information accompanies this paper on the Leukemia website (http://www.nature.com/leu) 